\title{
Frequency comb for THz metrology and spectroscopy
}

\author{
C. Bray ${ }^{1}$, A. Cuisset ${ }^{1}$, F. Hindle ${ }^{1}$, JF. Lampin ${ }^{2}$ and G. Mouret ${ }^{1}$ \\ ${ }^{1}$ Laboratoire de Physico-Chimie de l'Atmosphère, Université du Littoral Côte d'Opale, 189A Avenue Maurice Schumann, \\ 59140 Dunkerque, France, mouret@univ-littoral.fr \\ ${ }^{2}$ Institute of Electronics, Microelectronics and Nanotechnology, UMR 8520, University of Lille, Villeneuve d'Ascq, France
}

Frequency combs (FC) have radically changed the landscape of frequency metrology and highresolution spectroscopy investigations extending tremendously the achievable resolution while increasing signal to noise ratio. By describing two experiments, we highlight that like other spectral ranges, $\mathrm{THz}$ domain can exploit a frequency comb.

First, the photomixing experiment we have developed at the LPCA during the last decade is presented on figure 1. This spectrometer is able to work between 300 and $3000 \mathrm{GHz}$ with a frequency accuracy and a $\mathrm{THz}$ frequency linewidth suitable for high resolution $\mathrm{THz}$ spectroscopy [1-2]. The photomixing process is known from long time, and consist to convert an optical beat note into the $\mathrm{THz}$ domains. It is one of the best approach to cover a large part of the $\mathrm{THz}$ frequency gap. Thus, the frequency of the $\mathrm{THz}$ radiation produced is just the difference frequency of the two lasers working around $750 \mathrm{~nm}$ (and corresponding to a frequency around $400 \mathrm{THz}$ ) required to use of photomixer made in LTG GaAs. In principle, the frequency accuracy of the radiated $\mathrm{THz}$ radiation depends of the frequency accuracy of the pumps lasers. Clearly, there are no commercial solution to reach a precision better than $100 \mathrm{kHz}$ often required for high resolution rotational spectroscopy. An alternative way consists to use a FC produce by a femtosecond laser as a ruler to control and to measure the difference frequency between the two pump lasers. In the present demonstration, the FC is produced by a frequency doubled erbium doped modelocked fiber laser with a stabilized repetition rate around 100 $\mathrm{MHz}$. A beatnote between the laser and the nearest FC mode was isolated and phase locked to a local oscillator coherently locking the diode to the FC. The locking of the second laser to a different FC mode allows the difference frequency between the pump lasers to be synthesized. Feedback correction signal is applied to control the difference frequency of pump laser, and thus the $\mathrm{THz}$ frequency. The difference of frequency between the diode lasers can be expressed in Eq. (1) as:

$$
f_{T H z}=n \cdot f_{r r} \pm f_{s} \pm f_{s}
$$

where $f_{r r}$ is the repetition rate of the femtosecond laser, $n$ is an integer, and $f_{s}$ is the frequency of the synthesizer used to phase lock the beat signals against synthesizer to a precise frequency control. A classical commercial wavemeter is used to remove the ambiguity of the integer.
Various species have been study by use of this spectrometer from highly polar molecules (OCS, $\left.\mathrm{H}_{2} \mathrm{CO}\right)$ up to low and non-polar $\left(\mathrm{CH}_{3} \mathrm{D}, \mathrm{CH}_{4}\right)$ compounds and as well as radicals $(\mathrm{OH}, \mathrm{SO})$ to cite a fews [3-6].

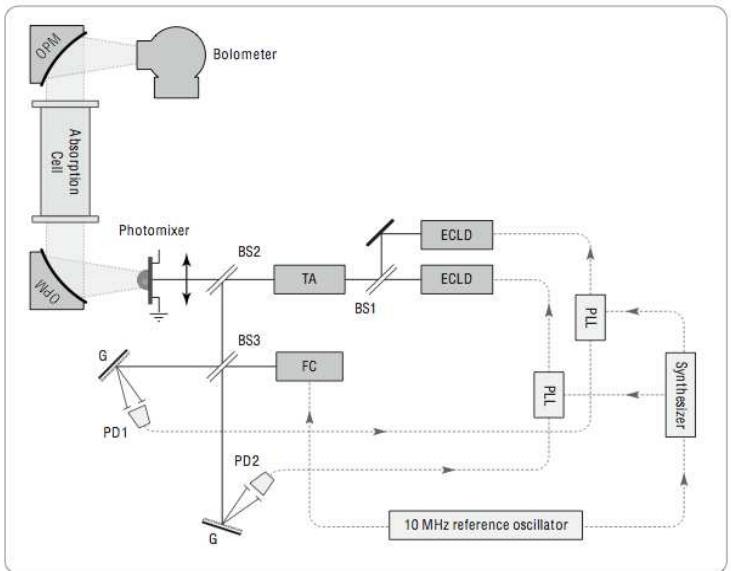

Fig. 1. Experimental setup of the $\mathrm{THz}$ photomixing spectrometer. BS1 is a beam splitter to overlap the two laser beams from the two External Cavity Diode Laser (ECDL) and seed Tapered Amplifier (TA). Then, the optical beat note is divided by a second beam splitter (BS2) to produce $\mathrm{THz}$ radiation and to manage the $\mathrm{THz}$ frequency. BS3 is used to overlap optical beat note and the frequency comb. Two grating $(\mathrm{G})$ disperse the FC and the two lasers to ensure a heterodyne analysis between each ECDL and a part of FC thank to photodiodes (PD1 and PD2). The heterodyne signals are used to control the frequency of each laser.

Second, a very popular approach for $\mathrm{THz}$ investigations is the well-known $\mathrm{THz}$ Time Domain Spectrometer. Except the dual comb approach, the discrete spectral properties of the generated radiation which forms a $\mathrm{THz}$ offset free frequency comb is often ignored. However, the exploitation of the frequency comb structure of $\mathrm{THz}$ pulses should lead to an exceptional frequency resolution. To explore this approach, a dedicated heterodyne detection has been implemented around $200 \mathrm{GHz}$ in order to analyse at very high resolution the $\mathrm{THz} \mathrm{FC}$ (figure 2). Knowing exactly the repetition rate of the femtosecond laser used to produce the $\mathrm{THz} F \mathrm{FC}$ and intermediate frequency thanks to spectrum analyser, different components of the THz FC can be identified without ambiguity (figure 3). By tuning the repetition rate and/or the frequency of the local oscillator, high resolution spectroscopy can be performed [7].

Those two examples show the potential of the Frequency Comb to improve spectroscopic investigations in the $\mathrm{THz}$ domain. 


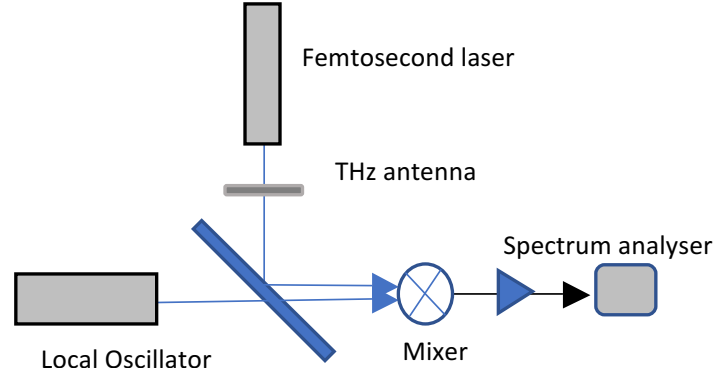

Fig. 2. Heterodyne spectrometer. The $\mathrm{THz} \mathrm{FC}$ is produced by focusing optical fs pulses onto a a photoconductive dipole antenna. The THz FC and the local oscillator (formed by an amplifier multiplier chain driven by a synthesizer) is overlapped by a beam splitter to feed a subharmonic mixer. After amplification, the intermediate frequency (IF) signal is recorded by a classical spectrum analyzer.

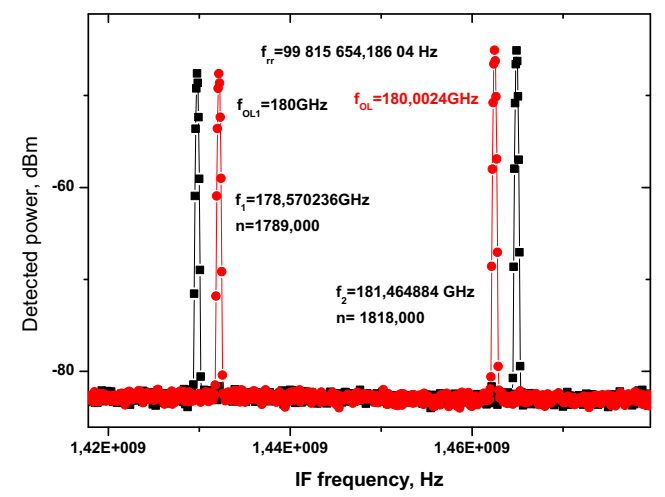

Fig. 3. High resolution spectra of the THZ FC obtained by use of heterodyne detection in which each component is clearly identified by an integer corresponding to an exact harmonic of the repetition rate.

\section{Acknowledgements}

This study was undertaken as part of the HEROES project which is financially supported by Agence Nationale pour la Recherche (project number ANR-16-CE30-0020).

\section{References}

1. G. Mouret, et al., $\mathrm{THz}$ photomixing synthesizer based on a fiber frequency comb // Optics Express, Vol. 17(24), 22031-22040 (2009)

2. F. Hindle, et al., Widely tunable $\mathrm{THz}$ synthesizer // Applied Physics B Vol. 104(4) 763-768 (2011)

3. S. Eliet, et al., Rotational spectrum of formaldehyde reinvestigated using a photomixing $\mathrm{THz}$ synthesizer // Journal of Molecular Spectroscopy, Vol. 279, 12-15 (2012)

4. M.A. Martin-Drumel, et al., New investigation on $\mathrm{THz}$ spectra of $\mathrm{OH}$ and $\mathrm{SH}$ radicals // Chemical Physics Letters, Vol. 550, 8 - 14 (2012)

5. C. Bray, et al., $\mathrm{CH}_{3} \mathrm{D}$ photomixing spectroscopy up to $2.5 \mathrm{THz}$ : New set of rotational and dipole parameters, first THz self-broadening measurements // Journal of Quantitative Spectroscopy \& Radiative Transfer, Vol. 189, 198205 (2017)

6. C. Bray, et al. Spectral lines of methane measured up to $2.6 \mathrm{THz}$ at sub-MHz accuracy with a CW-THz photomixing spectrometer: Line positions of rotational transitions induced by centrifugal distortion // Journal of Quantitative
Spectroscopy \& Radiative Transfer, Vol. 203, 349-354 (2017).

7. S. Skryl et al., High-resolution terahertz spectroscopy with a single tunable frequency comb //Opt. Optics Express, Vol. 22 (26), 32276-32281 (2014). 